\title{
Impaired arm activity in COPD: a questionable goal for rehabilitation
}

\author{
Enrico M. Clini ${ }^{1}$ and Nicolino Ambrosino ${ }^{2}$
}

Affiliations: 'Dept of Medical and Surgical Sciences, Ospedale Villa Pineta, University of Modena-Reggio Emilia, Modena, and ${ }^{2}$ Pulmonary Rehabilitation and Weaning Center, Auxilium Vitae, Volterra, Italy.

Correspondence: Nicolino Ambrosino, Pulmonary Rehabilitation and Weaning Center, Auxilium Vitae, Borgo San Lazzaro, 5-56048, Volterra, Italy. E-mail: nico.ambrosinodgmail.com

0 @ERSpublications

Arm training should be included in pulmonary rehabilitation programmes of COPD patients http://ow.ly/tkRVp

The most common extrapulmonary problems in chronic obstructive pulmonary disease (COPD) patients are cardiovascular comorbidities, muscle weakness and dysfunction [1-3]. Indeed, the reduced peripheral muscle mass and strength increase patient's symptoms during effort (fatigue and or dyspnoea) and progressively lead to disability. Furthermore, both lower and upper limb dysfunction has been shown to independently predict the long-term prognosis of COPD patients [2].

The use of arms is involved in many daily domestic tasks and activities. To date, it has been shown that daily arm activities needing elevation above the shoulders may interfere with regular breathing and are associated with dynamic hyperinflation in COPD patients [4]. The research by MeIJeR et al. [5], published in this issue of the European Respiratory Journal, investigated whether, and in which proportion of muscle effort, arm activities during daily life are different in COPD patients compared with matched healthy individuals. Notably, this study is the first to characterise arm activities associated with daily living in the COPD population.

Daily arm and leg domestic activities were measured by accelerometers in moderate-to-severe COPD patients and controls. After correcting for the daily walking time, the time spent on arm activities was similar in COPD patients and healthy controls, whereas the intensity of these activities was lower in COPD patients. In addition, the authors assessed the relative effort of the trapezius, deltoid and biceps muscles using electromyography during 12 standard domestic activities involving upper limbs in both populations. COPD patients performed seven arm activities at a lower intensity compared to control subjects, while the degree of arm elevation was similar. Moreover, in COPD patients compared with controls, muscle effort was found to be performed at a higher proportion of the maximum for the trapezius during six activities (writing on a board, shaking hands, folding towels, putting towels on the shelf, walking at preferred speed and sweeping the floor) and for the biceps during only one activity (folding towels). Overall, the total effort over the total effort spent by the trapezius muscle during these specific arm activities in COPD patients was roughly twice that required in subjects without COPD.

The findings by MeIJER et al. [5] challenge the anecdotal observation that arm activities may be problematic and poorly tolerated in patients suffering from COPD [6]. As a matter of fact, this study clearly indicates that domestic arm activities in COPD patients are performed differently from the point of view of intensity (less) but not time (same duration). Although mechanical efficiency and exercise capacity of the upper limb muscles are usually preserved in COPD patients, it is likely that repeated domestic arm activities may enhance their symptoms and ventilator constrains [7].

Received: Jan 032014 | Accepted: Jan 062014

Conflict of interest: None declared.

Copyright @ERS 2014 
In COPD patients, severity and impact of dyspnoea during living activities is relevant and, at least in part, may be due to the relationship between their reduced ventilatory capacity and physical deconditioning [7]. COPD patients have reduced peak aerobic capacity and experience more difficulty during domestic activities [8]. In fact, in moderate-to-severe COPD patients, raising unsupported arms above the shoulders increases oxygen uptake and minute ventilation above $50 \%$ of the maximum, thus leading to perceived worsening muscle fatigue and tiredness $[9,10]$.

In practice, patients may reduce intensity of their arm activities because these are often involved during daily living. Given the preserved overall time spent with these arm activities, COPD patients may either reduce the daily number of tasks involving arms or perform a similar number of tasks at a lower intensity [11].

The overall reduction of movement intensity when performing activities with the shoulder/arm muscles in COPD patients does not automatically translates into less relative effort of the single muscle mass. Indeed, biceps and deltoid muscles work at the same relative muscle effort as in healthy individuals, whereas the trapezius doubles its relative effort. The latter result might be due to the inspiratory function of the trapezius muscle, especially for those domestic activities, including walking, that are more likely to increase ventilator requirement and/or require arm elevation with associated loss of lung volume capacity [12].

The findings of impaired arm activities by MEIJER et al. [5] open the practical question as to whether or not arm training should be included as a useful and appropriate component in the rehabilitation course of COPD patients.

Recently, two controlled studies addressed this topic. Costi et al. [13] showed that unsupported arm training may result in a functional benefit greater than that achieved with standard rehabilitation. These authors have shown that specific arm performance may improve arm capacities, a result still maintained over 6 months. This is particularly important as maintenance of daily activities after rehabilitation is a difficult task for COPD patients [14]. JANUDIS-FERREIRA et al. [15] demonstrated that resistance-based arm training improved arm function, strength and exercise capacity although this did not translate into lower dyspnoea during domestic activities.

Taken together, these data would support the inclusion of an arm training programme in standard pulmonary rehabilitation programmes of COPD patients. Surprisingly, despite arm cycling being recommended in critically ill patients in the intensive care unit [16], the most up-to-date document on pulmonary rehabilitation proposed by the American Thoracic Society/European Respiratory Society, including also some of the authors of the present study [5], does not specifically suggest the inclusion of arm training in standard pulmonary rehabilitation programmes of patients suffering from chronic respiratory diseases [17]. Indeed, the two major problems in defining the exact role and contribution of arm training in COPD are still the appropriateness of methods to assess impairment, and the likely mechanisms by which training improves arm function and ability.

A recent systematic review underlined that there is growing interest in the assessment of upper limb exercise capacity in disabled COPD patients, in order to better document the impact of upper limb training [18]. Pragmatically, incremental arm ergometry may be the best method for measuring peak supported arm exercise capacity and endurance. However, sub-maximal tests such as the upper limb exercise test, 6-min pegboard and ring test, and grocery shelving tasks are more likely to reflect activities of daily living and, thus, should be the tests of choice to measure unsupported arm exercise capacity and function. Since limb motor dysfunction is detectable in $\sim 30 \%$ of COPD patients [19], this indicates the need to assess it early. The study by MeIJER et al. [5] has the merit to be the first to objectively introduce the assessment of a specific muscle performance when arms are involved during sub-maximal activities, thus giving a complementary view to characterise this functional aspect in the everyday life of COPD patients.

However, one might argue that improving muscle strength and intensity per se with training involving arms does not automatically mean that better skills with arm activities are achieved. We are aware of only one study evaluating the mechanisms through which unsupported arm muscle training might be beneficial to COPD patients [20]. In that physiological setting on patients tested at $80 \%$ of their peak incremental arm exercise, before and after training, the authors found that a reduced level of ventilation, relative to a decrease in central motor output, is the contribution of training to symptom alleviation, and that patients' improved exercise-related perception did not affect chest wall operational volumes. Therefore, rather than strengthening muscle mass, an "appropriate" adaptation of central motor output and ventilation to exercise might be advocated as the best physiological goal to be achieved in order to obtain practical benefits during domestic activities involving arms. Furthermore, it is also possible that different degrees of patient's disability (i.e. patients with reduced daytime arm activities), more severe peripheral muscle dysfunction, older age and coexisting chronic comorbidities may influence the ultimate effects and responses to arm training. This requires further studies for a more detailed characterisation of arm dysfunction and the related specific intervention in COPD individuals. 
To conclude, it seems at least reasonable to hypothesise that specific training may improve arm muscle capacity, reduce the task-related dyspnoea and finally decrease the burden of frequent or repetitive domestic activities. The actual body of evidence, despite inconclusive, warrants further research in this area aimed at improving the methods for assessment and characterisation of arm dysfunction, and at studying the most appropriate and tailored intervention with the ultimate goal to respond to a specific need in the COPD population.

\section{References}

1 Vanfleteren LE, Spruit MA, Groenen M, et al. Clusters of comorbidities based on validated objective measurements and systemic inflammation in patients with chronic obstructive pulmonary disease. Am J Respir Crit Care Med 2013; 187: 728-735.

2 Divo M, Cote C, de Torres JP, et al. Comorbidities and risk of mortality in patients with chronic obstructive pulmonary disease. Am J Respir Crit Care Med 2012; 186: 155-161.

3 Sibila O, Mortensen EM, Anzueto A, et al. Prior cardiovascular disease increases long-term mortality in COPD patients with pneumonia. Eur Respir J 2014; 43: 36-42.

4 Gigliotti F, Coli C, Bianchi R, et al. Arm exercise and hyperinflation in patients with COPD: effect of arm training. Chest 2005; 128: 1225-1232.

5 Meijer K, Annegarn J, Lima Passos V, et al. Characteristics of daily arm activities in patients with COPD. Eur Respir J 2014; 43: 1631-1641.

6 Annegarn J, Meijer K, Passos VL, et al. Problematic activities of daily life are weakly associated with clinical characteristics in COPD. J Am Med Dir Assoc 2012; 13: 284-290.

$7 \quad$ Vaes AW, Wouters EF, Franssen FM, et al. Task-related oxygen uptake during domestic activities of daily life in patients with COPD and healthy elderly subjects. Chest 2011; 140: 970-979.

8 Pitta F, Takaki MY, Oliveira NH, et al. Relationship between pulmonary function and physical activity in daily life in patients with COPD. Respir Med 2008; 102: 1203-1207.

9 Gosker HR, Lencer NH, Franssen FM, et al. Striking similarities in systemic factors contributing to decreased exercise capacity in patients with severe chronic heart failure or COPD. Chest 2003; 123: 1416-1424.

10 Velloso M, Garcia Stella S, Cendon S, et al. Metabolic and ventilatory parameters of four activities of daily living accomplished with arms in COPD patients. Chest 2003; 123: 1047-1053.

11 Velloso M, Jardim JR. Study of energy expenditure during activities of daily living using and not using body position recommended by energy conservation techniques in patients with COPD. Chest 2006; 130: $126-132$.

12 Marquis N, Debigare R, Bouyer L, et al. Physiology of walking in patients with moderate to severe chronic obstructive pulmonary disease. Med Sci Sports Exerc 2009; 41: 1540-1548.

13 Costi S, Crisafulli E, Antoni FD, et al. Effects of unsupported upper extremity exercise training in patients with COPD: a randomized clinical trial. Chest 2009; 136: 387-395.

14 Soicher JE, Mayo NE, Gauvin L, et al. Trajectories of endurance activity following pulmonary rehabilitation in COPD patients. Eur Respir J 2012; 39: 272-278.

15 Janaudis-Ferreira T, Hill K, Goldstein RS, et al. Resistance arm training in patients with COPD: a randomized controlled trial. Chest 2011; 139: 151-158.

16 Ambrosino N, Venturelli E, Vagheggini G, et al. Rehabilitation, weaning and physical therapy strategies in the chronic critically ill patients. Eur Respir J 2012; 39: 487-492.

17 Spruit MA, Singh SJ, Garvey C, et al. An official American Thoracic Society/European Respiratory Society statement: key concepts and advances in pulmonary rehabilitation. Am J Respir Crit Care Med 2013; 188: e13-64.

18 Janaudis-Ferreira T, Beauchamp MK, Goldstein RS, et al. How should we measure arm exercise capacity in patients with COPD? A systematic review. Chest 2012; 141: 111-120.

19 Carrai R, Scano G, Gigliotti F, et al. Prevalence of limb muscle dysfunction in patients with chronic obstructive pulmonary disease admitted to a pulmonary rehabilitation centre. Clin Neurophysiol 2012; 123: $2306-2311$.

20 Romagnoli I, Scano G, Binazzi B, et al. Effects of unsupported arm training on arm exercise-related perception in COPD patients. Respir Physiol Neurobiol 2013; 186: 95-102. 